\title{
Computational Analysis of Protein Structure Changes as a Result of Nondeletion Insertion Mutations in Human $\beta$-Globin Gene Suggests Possible Cause of $\beta$-Thalassemia
}

\author{
Talal Qadah ${ }^{1}{ }^{1}$ and Mohammad Sarwar Jamal $\mathbb{D}^{1,2}$ \\ ${ }^{1}$ Department of Medical Laboratory Technology, Faculty of Applied Medical Science, King Abdulaziz University, Jeddah, Saudi Arabia \\ ${ }^{2}$ Hematology Research Group, King Fahd Medical Research Center, King Abdulaziz University, Jeddah, Saudi Arabia \\ Correspondence should be addressed to Talal Qadah; thqadah@kau.edu.sa
}

Received 30 June 2018; Revised 28 February 2019; Accepted 11 April 2019; Published 29 May 2019

Academic Editor: Magdalena Kasprowicz

Copyright ( 2019 Talal Qadah and Mohammad Sarwar Jamal. This is an open access article distributed under the Creative Commons Attribution License, which permits unrestricted use, distribution, and reproduction in any medium, provided the original work is properly cited.

\begin{abstract}
Beta-thalassemia is described as a group of hereditary blood disorders characterized by abnormalities in the synthesis of beta chains of hemoglobin. These anomalies result in different phenotypes ranging from moderate to severe clinical symptoms to no symptoms at all. Most of the defects in hemoglobin arise directly from the mutations in the structural $\beta$-globin gene $(H B B)$. Recent advances in computational tools have allowed the study of the relationship between the genotype and phenotype in many diseases including $\beta$-thalassemia. Due to high prevalence of $\beta$-thalassemia, these analyses have helped to understand the molecular basis of the disease in a better way. In this direction, a relational database, named HbVar, was developed in 2001 by a collective academic effort to provide quality and up-to-date information on the genomic variations leading to hemoglobinopathies and thalassemia. The database recorded details about each variant including the altered sequence, hematological defects, its pathology, and its occurrence along with references. In the present study, an attempt was made to investigate nondeletion mutations in the HBB picked up from HbVar and their effects using the in silico approach. Our study investigated 12 nucleotides insertion mutations in six different altered sequences. These 12 extra nucleotides led to the formation of a loop in the protein structure and did not alter its function. It appears that these mutations act as 'silent' mutations. However, further in vitro studies are required to reach definitive conclusions.
\end{abstract}

\section{Introduction}

Hemoglobinopathies are genetic disorders caused by singlegene variations in the $\alpha$-like and $\beta$-like human globin gene clusters. These are the most common inherited disorders in humans with nearly $7 \%$ of the human population acting as carriers of the mutations in globin genes. Single nucleotide substitutions in the coding or regulatory regions of these genes can lead to varying degrees of defects in their expression [1]. The $H B B$ gene belongs to the $\beta$-globin gene cluster that encodes $\beta$-globin polypeptide. It is located in the short arm of chromosome 11 and contains two introns and three exons. Molecular defects in human $H B B$ may result in structural defects causing abnormality in hemoglobins, such as $\mathrm{HbS}, \mathrm{HbC}$, and $\mathrm{HbD}$, or may result in absence or reduced synthesis of $\beta$-globin chains causing $\beta$-thalassemia [2]. Mutations in the $H B B$ may involve substitution, deletion, or insertion of one or multiple nucleotides within the gene or its flanking regions resulting in anemia and low RBC production [3]. $\beta$ - Thalassemia is inherited as an autosomal recessive trait and its clinical manifestation can be divided as thalassemia major, intermedia, and thalassemia minor (trait) [4]. Some mutations in the $H B B$ lead to complete inactivation of the gene resulting in the absence of $\beta$-globin chains $\left(\beta^{0}\right)$, in turn, leading to the most severe form of thalassemia. Other mutations allow production of $\beta$-globin chains in varying proportions leading to $\beta^{+}$thalassemia. This case is most commonly found in the Middle East, Central Asia, Mediterranean countries, India, and southern China and in some parts of Africa and South America [5]. It is one of the 
most common genetic disorders caused by point mutations in Saudi Arabia causing variable phenotypic effects. These phenotypic severities may arise from defects in transcription, RNA processing, or translation of the HBB gene [6]. The most common mutations in most Arab-populated countries include IVSI-110(G>A), IVSI-1(G>A), IVSI-6(T>C), IVSII$1(\mathrm{G}>\mathrm{A})$, IVSI-5(G>C), codon 5(-CT), and codon $39(\mathrm{C}>\mathrm{T})$ [7].

Due to the high prevalence of variable phenotypes of thalassemia and striking heterogeneity of its molecular defects, various strategies were employed to investigate the molecular mechanisms of this disease. Due to recent advances in computational tools, in silico analysis has become one of the chosen methods to investigate links between genomic and resulting phenotypic characteristics in thalassemia. HbVar (http:/globin.cse.psu.edu/globin/hbvar/) is the oldest and most appreciated database of hemoglobin variants and thalassemia mutations established in 2001 [8]. It is a locus specific database, which was developed as a combined academic effort to keep a record of hemoglobin variants, new data entries, updates, and corrections. It provides high quality and upto-date information on the genomic variations, associated phenotypic and hematological effects, pathology, frequency of different mutations, ethnic prevalence, and references [9]. HbVar has become a primary resource for the research community working on globin proteins and for physicians dealing with patients with hemoglobinopathies, to help them with making proper diagnoses.

The objective of this study is to investigate the effects of insertion mutations in the $H B B$ exons using the in silico approach. We aimed to search the HbVar database to select sequences with uncharacterized insertion mutations and study their effects on the structure and function of $\beta$-globin protein.

\section{Methodology}

To perform this study, data from the HbVar database (http://globin.bx.psu.edu/hbvar) were used. Using this database, we identified uncharacterized $H B B$ sequences containing nondeletion mutations and picked them up for in silico investigation. The sequence of a wild $H B B$ (gene ID 3043) was taken as reference (https://www.ncbi.nlm.nih.gov/ gene/?term=3043). The potential 5' and $3^{\prime}$ sites in the gene sequences were identified using the Human Splicing Finder software [10]. These predictions were compared and multiple sequence alignment was performed using the available web server. An online translation tool (https://web.expasy.org/translate/) was used to translate the nucleotide sequences into amino acids, and the types of mutations as well as their respective positions were noted. Mutations located in the defined donor and acceptor splice sites were included here. The relative strength of the sites obtained from the bioinformatics tool was given values between 0 to 100 . Splice sites with high value were considered functional. Homology modeling of the wild type and mutant sequences was performed to compare the $3 \mathrm{D}$ structures of the proteins using the SWISS-MODELER [11].
TABLE 1: Variation in the protein sequences.

\begin{tabular}{lc}
\hline Amino Acids & Sequence_ID \\
\hline R31 & Seq1, Seq3, Seq 4, Seq5, Seq6 \\
S31 & Seq2 \\
L32 & Seq1, Seq2, Seq3, Seq6 \\
M32 & Seq5 \\
V32 & Seq4 \\
V33 & Seq1, Seq2, Seq3, Seq 4, Seq5 \\
I33 & Seq6 \\
\hline
\end{tabular}

\section{Molecular Dynamic Simulation}

The molecular dynamic simulation of the wild type and mutant proteins was performed using the GROMACS software [12]. The force field used for simulation was GROMOS96 53A6. The model structures (wild type and mutants) were solvated with water molecules in an octahedral box. Sodium ions $\left(\mathrm{Na}^{+}\right)$were added for neutralization. The solvated systems were then subjected to 5000 steps of energy minimization using the steepest descent method to remove the steric clashes. Convergence was achieved in the energy minimization when the maximum force was smaller than $1000 \mathrm{~kJ} \mathrm{~mol}^{-1} \mathrm{~nm}^{-1}$. The NPT ensemble was performed for $1000 \mathrm{ps}$ at $300 \mathrm{~K}$. The production simulation was executed at $300 \mathrm{~K}$ for $30 \mathrm{~ns}$ for the wild type and mutant proteins. Protein visualization and superimposition were performed using PyMOL software (https://pymol.org/2/). Root mean square deviation was analyzed using the PyMOL align module.

\section{Results}

Multiple sequence alignment between reference and mutated gene sequences showed that at least 12 nucleotides have been inserted in the sequences $1,2,3,4,5$, and 6 from position 93 to 104 as compared to the wild type (Figure 1). The inserted nucleotides showed maximum variation in the positions 93 and 94 . In sequence 1 and sequence 2 , adenine and thymine were inserted at position 93, respectively, whereas guanine appeared at position 93 in sequences 3, 4, 5, and 6. At position 94 , thymine was inserted in sequences 1,2 , and 6 , cytosine in sequence 3 , guanine in sequence 4 , and adenine in sequence 5. From position 95 to 104 , the six sequences presented no variation among the inserted nucleotides, except for sequence 6 which had adenine inserted at position 97, instead of guanine like the remaining sequences.

Variations among these inserted nucleotides gave rise to changes in amino acids in protein sequences. Twelve nucleotides in total gave rise to four amino acids where different variants have been identified (Figure 2 and Table 1).

The construction of the 3D structure of the HBB protein from the given mutated sequences showed that the inserted amino acids formed a loop structure (secondary structure) in the protein. The homology modeling of the wild type and mutant type $\mathrm{HBB}$ protein showed that the mutated segment did not form any well-defined secondary structure. 


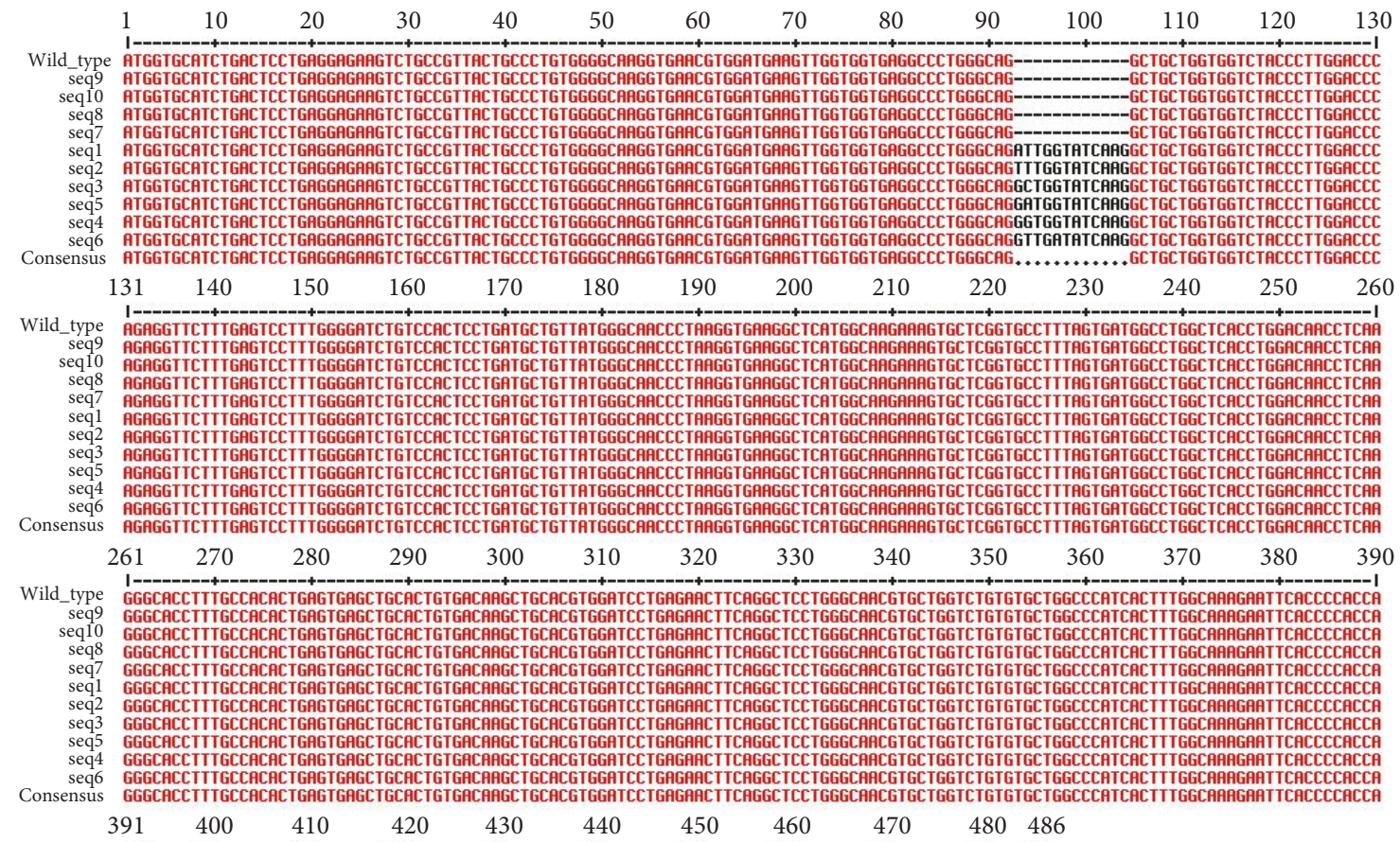

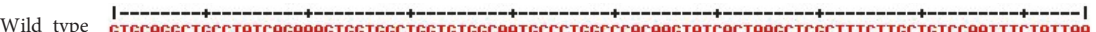

seq9 GTGCAGGCTGCCTATCAGAARGTGGTGGCTGGTGTGGCAATGCCCTGGCCCACARGTATCACTAAGCTCGCTTTCTTGCTGTCCAATTTCTATTA

seq10 GTGCAGGCTGCCTATCAGAAAGTGGTGGCTGGTGTGGCAATGCCCTGGCCCACAAGTATCACTAFGCTCGCTITCTTGCTGTCCAATTTCTATTAA

seq8 GTGCAGGCTGCCTATCAGAARGTGGTGGCTGGTGTGGCAATGCCCTGGCCCACAAGTATCACTAAGCTCGCTTTCTTGCTGTCCAATTTCTATTAP

seq7 GTGCAGGCTGCCTATCAGAARGTGGTGGCTGGTGTGGCARTGCCCTGGCCCACAAGTATCACTARGCTCGCTITCTTGCTGTCCAATTTCTATTAA

GTGCAGGCTGCCTATCAGAAAGTGGTGGCTGGTGTGGCAATGCCCTGGCCCACAAGTATCACTAAGCTCGCTTTCTTGCTGTCCARTITCTATTAP

seq2 3 GTGCAGGCTGCCTATCAGAAGGTGGTGGCTGGTGTGGCAATGCCCTGGCCCACAAGTATCACTAAGCTCGCTTTCTTGCTGTCCAATTTCTATTAA

seq5 GTGCAGGCTGCCTATCAGARAGTGGTGGCTGGTGGGGCARTGCCCTGGCCCACAAGTATCACTARGCTCGCTITCTTGCTGTCCARTTTCTATTAA

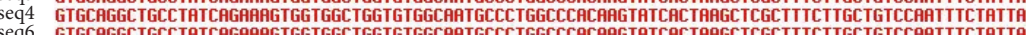

Consensus GTGCAGGCTGCCTATCAGAPRGTGGTGGCTGGTGTGGCARTGCCCTGGCCCACARGTATCACTARGCTCGCTTTCTTGCTGTCCAATTTCTATTAR

FIGURE 1: Multiple sequence alignment of the reference sequence and the multiple sequences, ATC, ATT, ACT, picked up from the HbVar database (after removing the introns). Twelve nucleotides have been inserted from position 93 to 104 in sequence numbers $1,2,3,4,5$, and 6 .

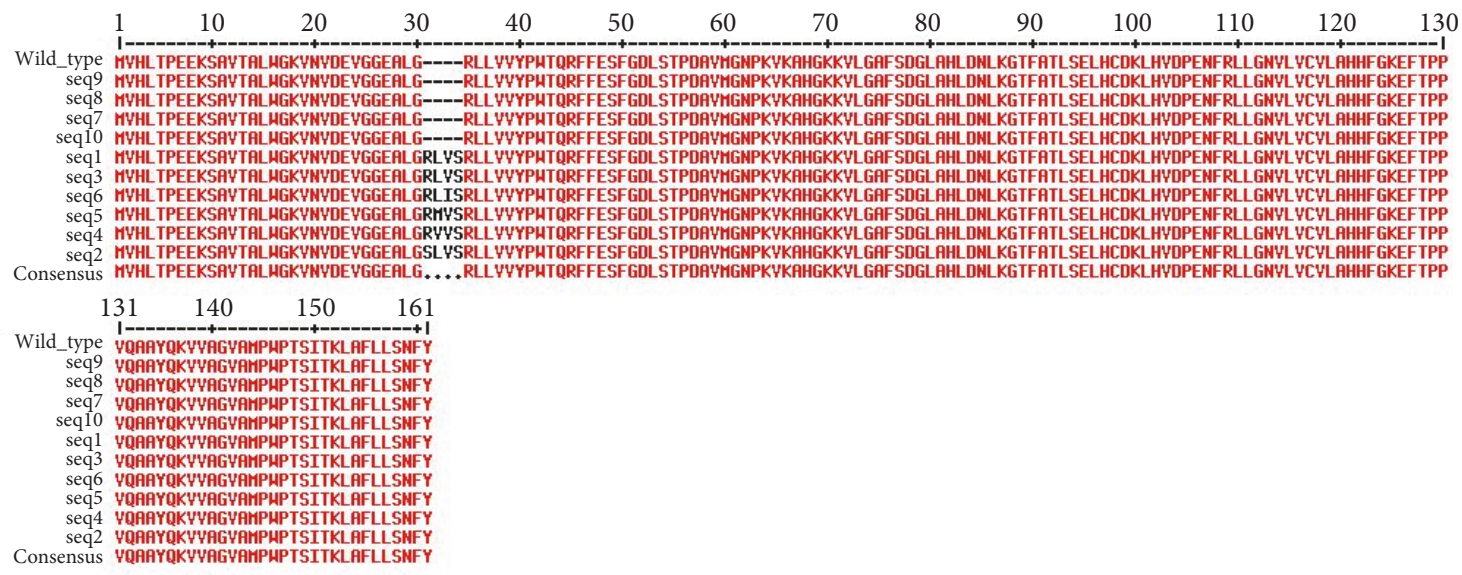

FIGURE 2: Protein sequence alignment of wild type and multiple sequences. Insertion of four amino acids has taken place at positions 31 to 34.

The mutated segment formed a loop and connected two $\alpha$ helical chains (Figure 3).

The stability and properties of the wild type and its mutant structures were studied by explicit solvent MD stimulation. Root mean square deviations have been calculated between different structures to form a complete picture of deviation in the structures of mutated proteins from the wild type (Table 2). The root mean square deviation (RMSD) analysis not only reflects the change of protein backbone versus simulation time, but also indicates the divergence of two structures. The RMSD of the homolog became stable at 30ns. The RMSD value of the wild type was $0.27 \mathrm{~nm}$ (Figure 4(a)). 

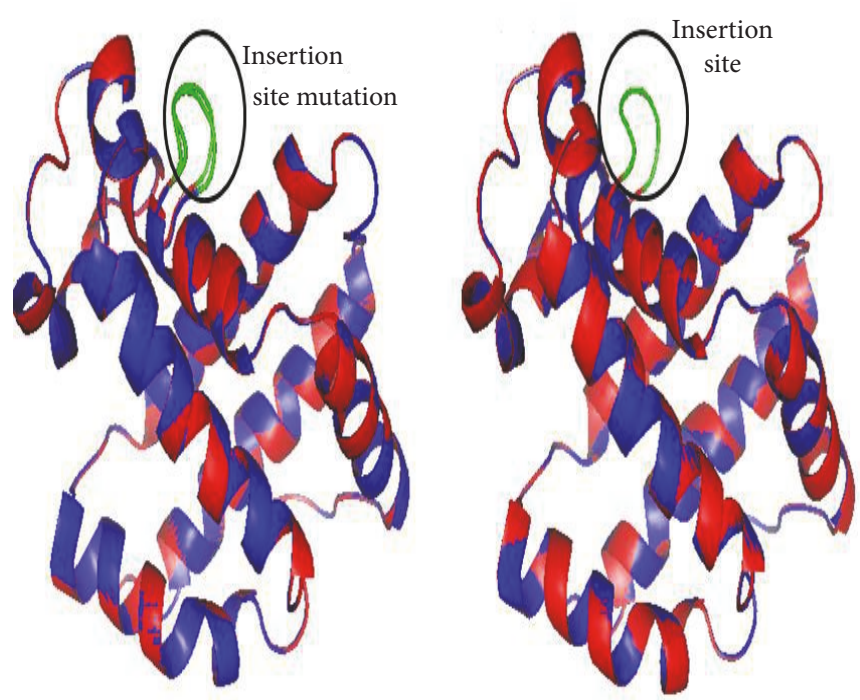

FIGURE 3: 3D structures of wild type (red color) and mutant type (blue color) of the HBB protein. Insertion sequence has been differentiated in green in the provided figures.

TABLE 2: Root mean square deviation (RMSD) between the model structures of the sequences.

\begin{tabular}{|c|c|c|c|c|}
\hline Model & Model 2 & RMS & Sec. str. & Mutation \\
\hline \multirow[t]{6}{*}{ WILD } & Seq1 & 1.115 & Loop & Insertion \\
\hline & Seq2 & 0.690 & Loop & Insertion \\
\hline & Seq3 & 1.115 & Loop & Insertion \\
\hline & Seq4 & 0.533 & Loop & Insertion \\
\hline & Seq5 & 0.534 & Loop & Insertion \\
\hline & Seq6 & 1.107 & Loop & Insertion \\
\hline \multirow[t]{5}{*}{ Seq2 } & Seq1 & 0.246 & Loop & \\
\hline & Seq3 & 0.246 & Loop & \\
\hline & Seq4 & 0.247 & Loop & \\
\hline & Seq5 & 0.256 & Loop & \\
\hline & Seq6 & 0.135 & Loop & \\
\hline Seq5 & Seq4 & 0.013 & Loop & \\
\hline
\end{tabular}

This result indicated that an accepted structure was obtained by the simulation that was reliable for further analyses. The root mean square fluctuation (RMSF) reflects the mobility of a certain residue around its mean position, which is another tool for studying the dynamics stability of the system. Although there were some deviations among the trajectories (especially in the loop region), the present data suggested less fluctuations, which further highlighted the reliability of the modeled structure (Figure 4(b)). The RMSF analysis can be used as a tool to describe local flexibility differences among residues throughout the $\mathrm{MD}$ simulation 62. According to Figure 4(b), the wild type protein and Seq6 showed an overall higher degree of flexibility when compared to the mutants. A difference in RMSF value was seen on residues 76-91. The wild type and Seq 6 proteins showed a fluctuation of $0.32 \mathrm{~nm}$, while the fluctuation at the same position on Seq1 was $0.1 \mathrm{~nm}$, thus indicating a flexibility loss. Whereas residues 54-80 showed fluctuation values ranging from $0.15 \mathrm{~nm}$ to $2.0 \mathrm{~nm}$ in the wild type, while in the Seq1 and Seq6, these fluctuation values ranged from $0.09 \mathrm{~nm}$ to $0.10 \mathrm{~nm}$. These results suggested that Seq1 affected the flexibility of the protein. This flexibility loss may affect protein function. Surprisingly, these fluctuations were found in the region away from the insertion or mutation site. However, Seq6 was found to have some fluctuation at this site as compared to the wild type and Seq1 (Figure 4(b)). We also analyzed the radius of gyration $(\mathrm{Rg})$ values in the simulation. $\mathrm{Rg}$ is an indicator of structure compactness and overall dimension of the protein. It explains how regular secondary structures are compactly packed into the $3 \mathrm{D}$ structure of a protein. If a protein is folded well, it will likely maintain a relatively steady value of $\mathrm{Rg}$, whereas it will change over time for unfolded proteins [13]. We found a stable $\mathrm{Rg}$ for Seq1 as compared to the wild type and Seq6 (Figure 4(c)). Low value of Rg for Seq1, as compared to the wild type and Seq6, 


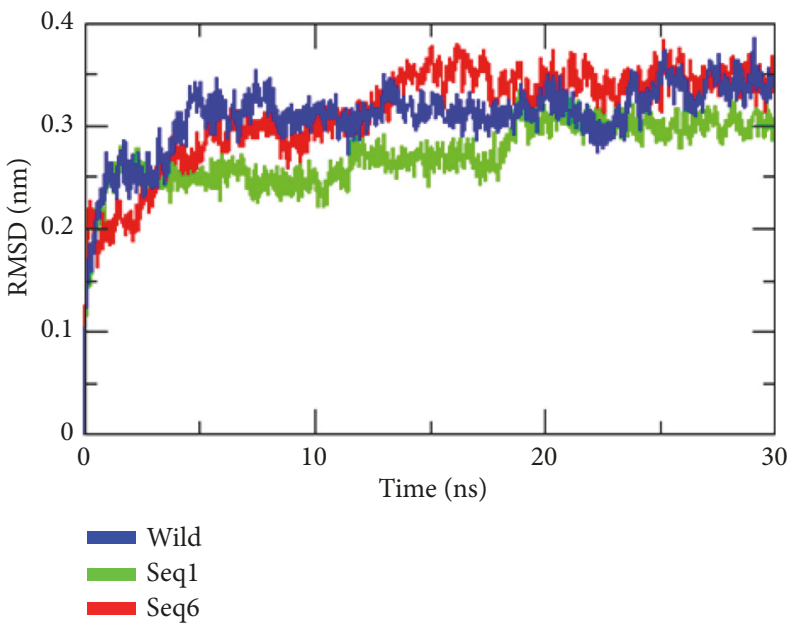

(a)

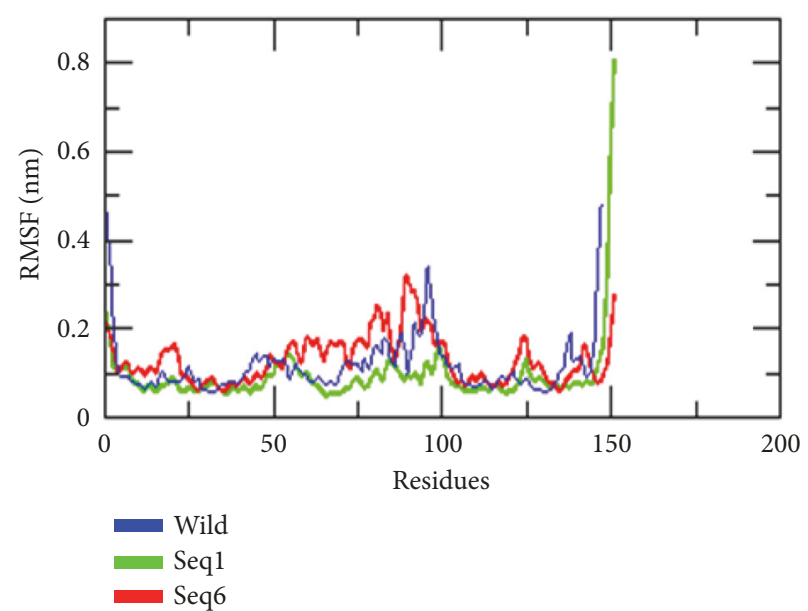

(b)

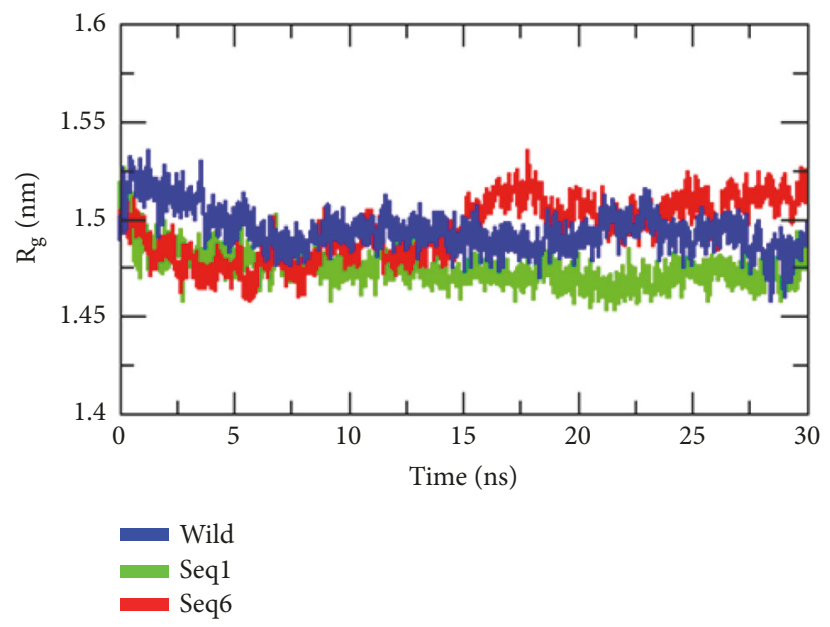

(c)

FIGURE 4: Molecular dynamic simulation: (a) root mean square deviation; (b) root mean square fluctuations; (c) radius of gyration of C $\alpha$ atoms of wild type and mutant proteins; time at $300 \mathrm{~K}$.

suggested tight packing of these structures, making them relatively stable. The conformation of the modeled structure of Seq1 with different times, from 10ns, 20ns, and 30ns, was found to be similar. However, a slight difference has been observed at the insertion site (Figure 5).

\section{Discussion}

Constructing a relationship between the genotype and phenotype experimentally is an important aspect of research [14], but it can prove to be highly difficult, in particular, when studying a large number of subjects. The in silico analysis provides a solution here. It helps researchers analyze enormous amounts of data in biology to narrow down the positive leads that can be further analyzed experimentally for validation. This saves an extensive amount of labor, time, and costs. In silico analysis of large number of mutations is also easier and faster to accomplish, as this type of investigation is performed by comparing and studying alterations in the nucleotide and/or amino acid sequences with the wild/native type and then correlating these alterations with the changed phenotypes $[15,16,16]$.

Generally, $\beta$-thalassemia affects the people of the Gulf, Middle Eastern, and Mediterranean regions [17]. Especially in Saudi Arabia, $\beta$ - thalassemia is prevalent, though there are variations in the frequency of the gene and in the type of mutations [18]. Many previous studies have screened and reported different mutations in the $H B B$ and their frequencies in the Saudi population [19-21].

Most of the mutations affecting the expression of the $H B B$ are linked to the gene physically and form different alleles of the gene, but some mutations that affect the gene expression, while also segregating the $H B B$ cluster, have also been identified [22, 23]. Although the $H B B$ is well characterized, some mutations in this gene recorded in the HbVar database are poorly understood and have not been properly studied before. In this study, we picked up such mutations and performed in silico analysis to understand their effect on the protein structure and function. 

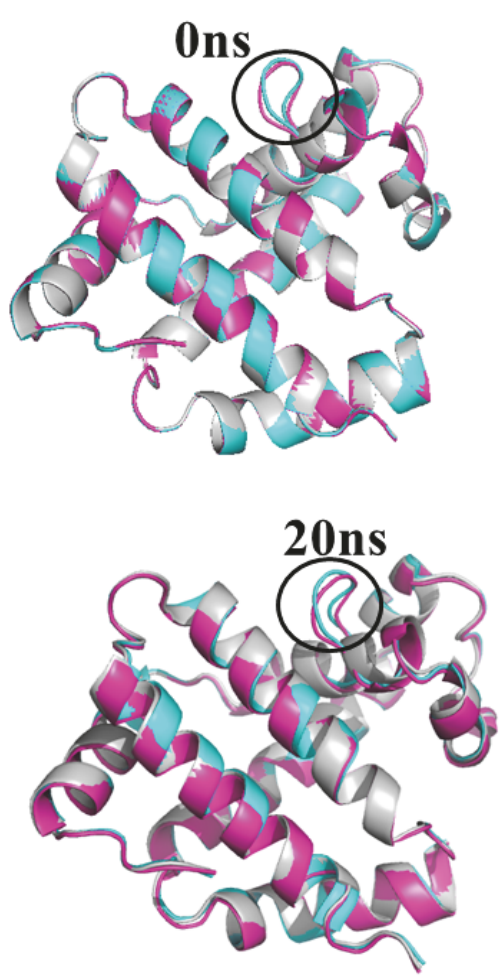
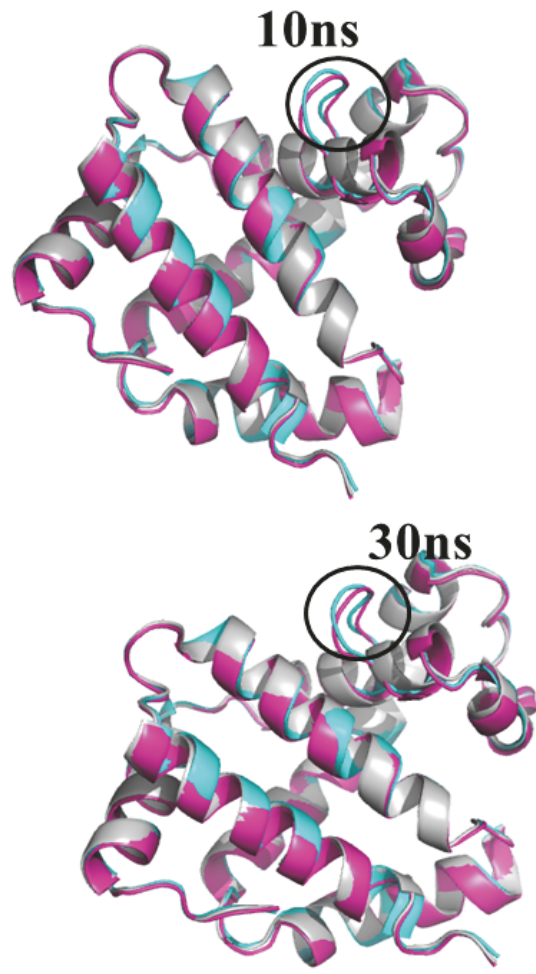

Figure 5: Conformation of model structures at 0ns, 10ns, 20ns, and 30ns. Cyan (Seq1), Magenta (Seq2), and Black brown (wild).

The nondeletion forms of defects in the $H B B$ account for a large number of the $\beta$-thalassemia alleles [24]. These include small insertions and single nucleotide substitutions or deletions within the gene or its nearby sequences [23]. Some of the alleles of $\beta$-thalassemia are very mild, in that carriers (heterozygotes) of such alleles are almost normal with no apparent signs of the disease, except for imbalanced synthesis of globin chains $[25,26]$. One of the fairly common 'silent' mutations in the Mediterranean population is 101 $\mathrm{C} \longrightarrow \mathrm{T}$. It interacts with other more severe mutations of $\beta$-thalassemia to cause milder forms [24]. Other 'silent' mutations have been reported in the 5' UTR region of the $H B B[25,26]$.

The present study aimed to understand the nondeletion mutations using in silico tools. We identified 6 different sequences carrying insertion mutation of 12 nucleotides from position 93 to 104 in the gene sequence. Variations also occurred among the inserted nucleotides among these 6 different sequences. In silico analysis showed that these inserted nucleotides translated into 4 additional amino acids. These additional amino acids acquired a loop formation in the 3D structure of the protein (Figure 3). The difference in amino acids did not show any variation in the secondary loop structure acquired by these amino acids, but the molecular dynamics simulations presented evidence of effects caused by these mutations on the overall protein flexibility. The RMSF analysis showed a high degree of flexibility in the wild type protein and Seq6 in comparison to other mutant forms (Figure 4(b)). Additionally, significant flexibility loss was seen in the Seq1 mutant form, especially in residues from positions 54-80 and 76-91. Though this effect was seen far from the site of mutation in Seq1, it is quite possible that insertion of new amino acids may likely disturb the internal environment of the protein, resulting in a whole new set of interactions between amino acids, which in turn, might have affected protein flexibility. This loss in flexibility may result in the loss of function of the protein. Studies suggest that a change in protein structure and, consequently, in function could be because of genetic variation in distal effect, because of the temporal effect due to folding of the protein sequence culminating into the final protein structure. This particularly happens because of the change in the properties related to the physicochemical, such as hydrophobicity, charge, and geometry due to the side chain of the amino acid residues. If such changes occur at critical sites, such as catalytic positions or important interacting sites called interfaces, then it is likely the reason for disease causing variations, which further tend to destabilize various hydrogen bonds and the salt bridge [2528]. To further prove the effects of these insertion mutations on protein structure and function, more in depth analysis is required.

Furthermore, the studies on radius of gyration of $\mathrm{C} \alpha$ atoms of the wild type and mutant proteins depicted in the $3 \mathrm{D}$ structure of the Seq1 mutant protein, are seen to be more compact and stable, as compared to the wild type and Seq6 proteins. This data suggests that the insertion mutations in $\mathrm{HBB}$ protein might be affecting its overall structure and function as shown in Seq1 and Seq6, but more 
intensive studies are required to fully understand the scope of these effects. We are yet to determine how, if at all, these mutations affect the flexibility of $\mathrm{HBB}$ protein and whether this loss affects protein function and to what extent. In vitro studies will further assess the functional behavior of mutated proteins.

\section{Data Availability}

The data used to support the findings of this study are included within the article.

\section{Conflicts of Interest}

The authors have no conflicts of interest.

\section{Acknowledgments}

This project was funded by the Deanship of Scientific Research (DSR) at King Abdulaziz University, Jeddah, under grant no. J-726-142-38. The authors, therefore, acknowledge with thanks DSR for technical and financial support. The authors also would like to thank Prof. Abdulwahab Noorwali for providing the authors with his technical support.

\section{References}

[1] D. J. Weatherall, "Hemoglobinopathies worldwide: present and future," Current Molecular Medicine, vol. 8, no. 7, pp. 592-599, 2008.

[2] D. J. Weatherall and J. B. Clegg, The Thalassaemia Syndromes, Blackwell Science, Oxford, UK, 2001.

[3] R. Galanello and R. Origa, "Beta-thalassemia," Orphanet Journal of Rare Diseases, vol. 5, article 11, 2010.

[4] A. S. Warsy, M. A. ElHazmi, A. K. Al Momin, A. AlHazmi, and A. Aleem, "Extensive polymorphisms in saudi beta thalassaemia patients," Biosciences, Biotechnology Research Asia, vol. 10, no. 1, pp. 127-132, 2013.

[5] L. Zahed, "The Spectrum of beta-Thalassemia Mutations in the Arab Populations," Journal of Biomedicine and Biotechnology, vol. 1, no. 3, pp. 129-132, 2001.

[6] M. H. Qari et al., "Regional consensus opinion for the management of Beta thalassemia major in the Arabian Gulf area," Orphanet Journal of Rare Diseases, vol. 8, p. 143, 2013.

[7] B. Giardine, S. van Baal, P. Kaimakis et al., "HbVar database of human hemoglobin variants and thalassemia mutations: 2007 update," Human Mutation, vol. 28, no. 2, p. 206, 2007.

[8] F. O. Desmet, D. Hamroun, M. Lalande, G. Collod-Bëroud, M. Claustres, and C. Béroud, "Human splicing finder: an online bioinformatics tool to predict splicing signals," Nucleic Acids Research, vol. 37, no. 9, article e67, 2009.

[9] T. Schwede, J. Kopp, N. Guex, and M. C. Peitsch, "SWISSMODEL: an automated protein homology-modeling server," Nucleic Acids Research, vol. 31, no. 13, pp. 3381-3385, 2003.

[10] B. Hess, C. Kutzner, D. van der Spoel, and E. Lindahl, "GRGMACS 4: algorithms for highly efficient, load-balanced, and scalable molecular simulation," Journal of Chemical Theory and Computation, vol. 4, no. 3, pp. 435-447, 2008.
[11] P. Rogerson and G. A. Arteca, "Molecular size scaling in families of protein native folds," Journal of Mathematical Chemistry, vol. 49, no. 8, pp. 1493-1506, 2011.

[12] T. Carlice-dos-Reis, J. Viana, F. C. Moreira et al., "Investigation of mutations in the HBB gene using the 1,000 genomes database," PLoS ONE, vol. 12, no. 4, 2017.

[13] R. Dibyajyoti, "In silico analysis of $\beta$-thalassemia mutations in india and its neighbouring south east asian countries," Journal of Pharmaceutical, Chemical and Biological Sciences, vol. 6, no. 3, pp. 188-198, 2018.

[14] K. M. K. De Vooght, R. V. Wijk, and W. W. Van Solinge, "Management of gene promoter mutations in molecular diagnostics," Clinical Chemistry, vol. 55, no. 4, pp. 698-708, 2009.

[15] S. AbdulAzeez and J. F. Borgio, "In-silico computing of the most deleterious nsSNPs in HBA1 gene," PLoS ONE, vol. 11, no. 1, p. e0147702, 2016.

[16] T. Jalil, "IN-silico assessment of common $\beta$-globin gene mutations found in Khyber Pakhtunkhwa, Pakistan," Pakistan Journal of Physiology, vol. 14, no. 3, pp. 68-73, 2018.

[17] A. Al-Sultan, S. Phanasgaonkar, A. Suliman, M. Al-Baqushi, Z. Nasrullah, and A. Al-Ali, "Spectrum of $\beta$-thalassemia mutations in the eastern province of Saudi Arabia," Hemoglobin, vol. 35, no. 2, pp. 125-134, 2011.

[18] F. H. Hasounah, S. A. Sejeny, J. A. Omer, J. M. Old, and R. W. A. Oliver, "Spectrum of $\beta$-thalassaemia mutations in the population of saudi arabia," Human Heredity, vol. 45, no. 4, pp. 231-234, 1995.

[19] M. S. Akhtar, F. Qaw, J. Francis Borgio et al., "Spectrum of $\alpha$-thalassemia mutations in transfusion-dependent $\beta$ thalassemia patients from the eastern province of Saudi Arabia," Hemoglobin, vol. 37, no. 1, pp. 65-73, 2013.

[20] A. M. Abuzenadah, I. M. R. Hussein, G. A. Damanhouri et al., "Molecular basis of $\beta$-thalassemia in the western province of Saudi Arabia: Identification of rare $\beta$-thalassemia mutations," Hemoglobin, vol. 35, no. 4, pp. 346-357, 2011.

[21] A. K. Al-Ali, S. Al-Ateeq, and B. W. Imamwerdi, "Molecular bases of $\beta$-thalassemia in the Eastern province of Saudi Arabia," Journal of Biomedicine and Biotechnology, vol. 2005, no. 4, pp. 322-325, 2005.

[22] S. L. Thein and W. G. Wood, "The molecular basis of $B$ thalassemia, $\mathrm{d} B$ thalassemia, and hereditary persistence of fetal hemoglobin," in Disorders of Hemoglobin: Genetics, Pathophysiology, M. H. Steinberg, B. G. Forget, D. R. Higgs, and R. L. Nagel, Eds., pp. 323-356, Cambridge University Press, Cambridge, UK, 2009.

[23] S. L. Thein, "The molecular basis of $\beta$-thalassemia," Cold Spring Harbor Perspectives in Medicine, vol. 3, no. 5, Article ID a011700, 2013.

[24] J. M. Gonzalez-Redondo, T. A. Stoming, A. Kutlar et al., "A $\mathrm{C} \longrightarrow \mathrm{T}$ substitution at $\mathrm{nt}-101$ in a conserved DNA sequence of the promotor region of the $\beta$-globin gene is associated with 'silent' $\beta$-thalassemia," Blood, vol. 73, no. 6, pp. 1705-1711, 1989.

[25] E. Maragoudaki, E. Kanavakis, J. Traeger-Synodinos et al., "Molecular, haematological and clinical studies of the $-101 \mathrm{C}$ $\longrightarrow \mathrm{T}$ substitution of the $\beta$-globin gene promoter in $25 \beta$ thalassaemia intermedia patients and 45 heterozygotes," British Journal of Haematology, vol. 107, no. 4, pp. 699-706, 1999.

[26] A. David and M. J. E. Sternberg, "The contribution of missense mutations in core and rim residues of protein-protein interfaces to human disease," Journal of Molecular Biology, vol. 427, no. 17, pp. 2886-2898, 2015. 
[27] M. Petukh, T. G. Kucukkal, and E. Alexov, "On human diseasecausing amino acid variants: Statistical study of sequence and structural patterns," Human Mutation, vol. 36, no. 5, pp. 524534,2015

[28] Y. Yang, B. Chen, G. Tan, M. Vihinen, and B. Shen, "Structurebased prediction of the effects of a missense variant on protein stability," Amino Acids, vol. 44, no. 3, pp. 847-855, 2013. 


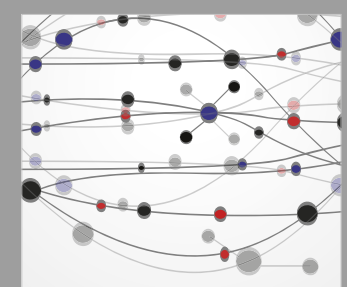

The Scientific World Journal
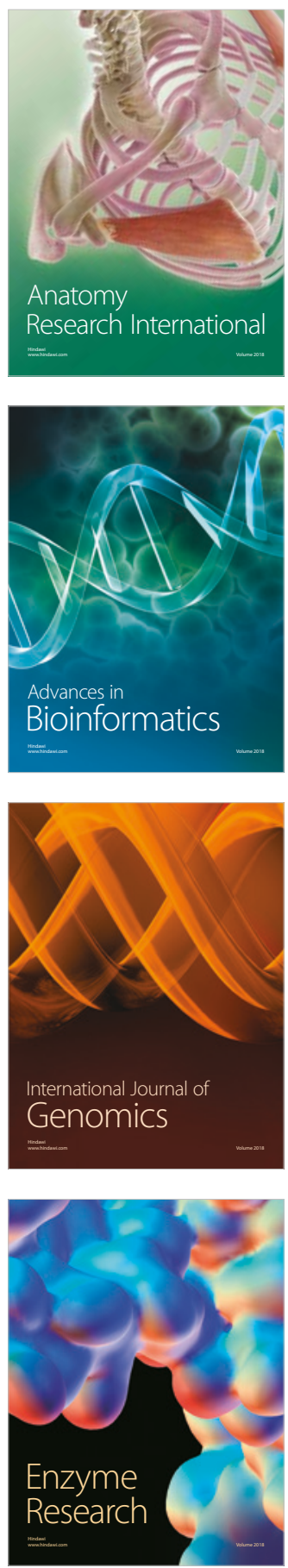
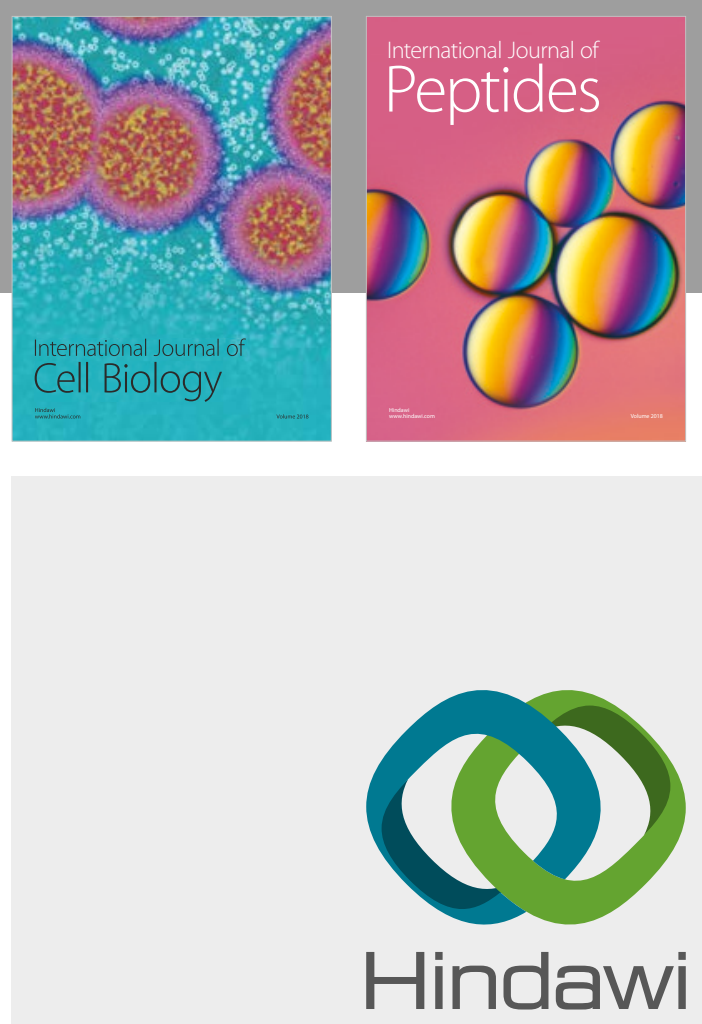

Submit your manuscripts at

www.hindawi.com
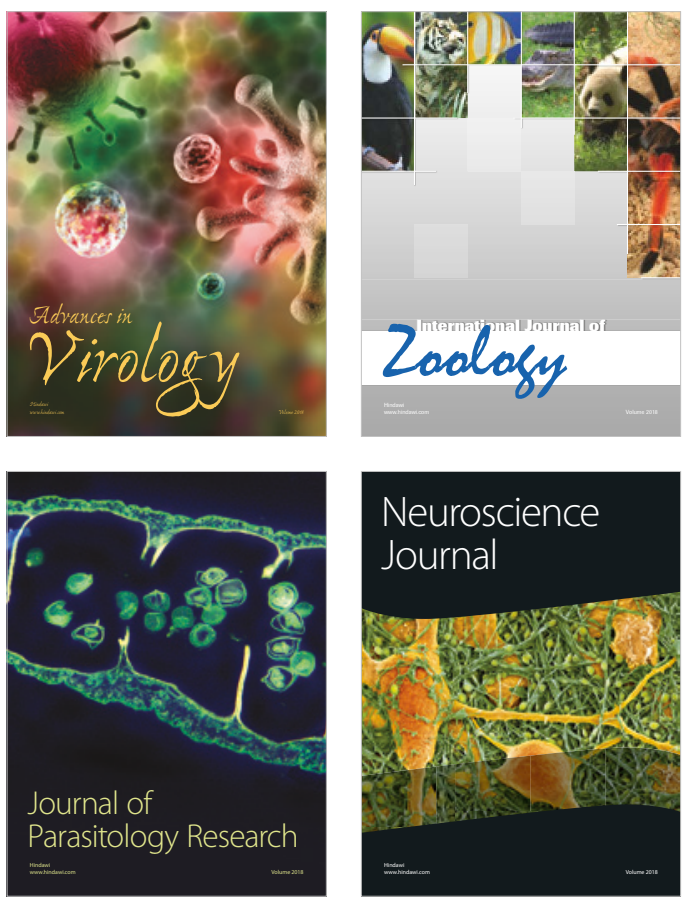
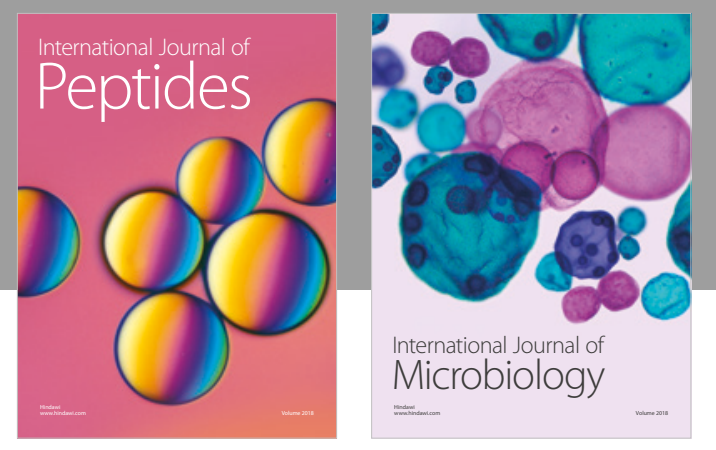

nternational Journal of Microbiology
Journal of
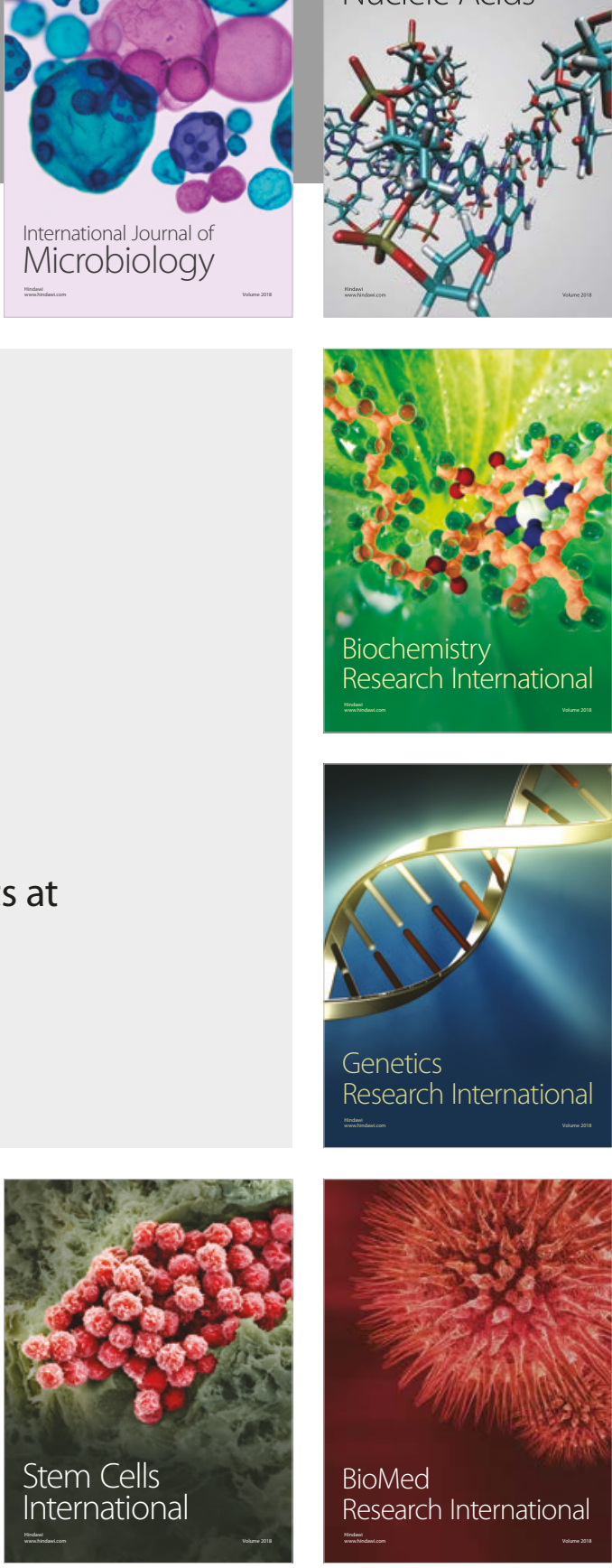
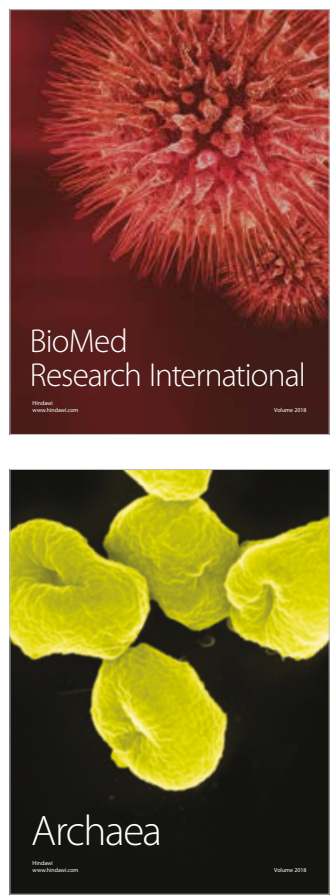